\title{
APPENDIX \\ Crossover Can Simulate Bounded Tree Search on a Fixed-Parameter Tractable Optimization Problem
}

\author{
Andrew M. Sutton \\ University of Minnesota Duluth
}

\section{PROOF OF LEMMA 4.4}

We define the orthogonal subcode of $\mathscr{C}(\ell)$ with respect to any $z \in$ $\mathbb{R}^{k}$ as

$$
\mathscr{C}_{\boldsymbol{z}}^{\perp}(\ell):=\left\{c_{i} \in \mathscr{C}(\ell): \sum_{j=1}^{k} c_{i}[j] z[j]=0\right\},
$$

i.e., the set of all code words in $\mathscr{C}(\ell)$ that are orthogonal to $z$ in $\mathbb{R}^{k}$.

Let $x \in \mathbb{R}^{k}$ be an arbitrary real-valued nonzero vector. We proceed by induction on $|\operatorname{supp}(\boldsymbol{x})|$. We decompose $\boldsymbol{x}$ into a linear combination $\boldsymbol{x}=\boldsymbol{y}+\boldsymbol{z}$ where $|\operatorname{supp}(\boldsymbol{y})|=|\operatorname{supp}(\boldsymbol{x})|-1$ and $|\operatorname{supp}(z)|=1$. Set $a \in[k]$ to the unique nonzero index in $z$. Choose $b \in \operatorname{supp}\left(B_{\ell}(a)\right)$ arbitrarily (note that $a \neq 0$ as $0 \notin[k]$ so such a choice is always possible).

We define a map

$$
\phi_{b}:[k] \rightarrow[(k+1) / 2-1] \cup\{0\}:=i \mapsto B_{\ell-1}^{-1}\left(B_{\ell}(i)[[\ell] \backslash\{b\}]\right) .
$$

Intuitively, $\phi_{b}(i)$ is the index element in $[(k+1) / 2-1]$ obtained by removing the bit in the $b$-th position of the length- $\ell$ bitstring representation of $i$.

We consider the orthogonal subcode $\mathscr{C}_{z}^{\perp}(\ell)$ with respect to $z$ and argue that the simplex code $\mathscr{C}(\ell-1)$ is isomorphic to $\mathscr{C}_{z}^{\perp}(\ell)$ in the following sense. For each $i, j \in[k]$, if $c_{i} \in \mathscr{C}_{\boldsymbol{z}}^{\perp}(\ell)$ and $B_{\ell}(j)[b]=0$, then

$$
c_{i}[j]=c_{\phi_{b}(i)}\left[\phi_{b}(j)\right] .
$$

In other words, the code words of $\mathscr{C}(\ell-1)$ can be recovered by taking each length- $k$ code word in $\mathscr{C}_{\boldsymbol{z}}^{\perp}(\ell)$ and deleting the elements in positions $j$ such that $B_{\ell}(j)[b]=1$. To prove this, note that under these conditions on $i$ and $j$,

$$
\begin{aligned}
c_{i}[j] & =\sum_{q=1}^{\ell} B_{\ell}(i)[q] B_{\ell}(j)[q] \quad(\bmod 2) \\
& =\left(\sum_{q \in[\ell] \backslash\{b\}} B_{\ell}(i)[q] B_{\ell}(j)[q]+B_{\ell}(i)[b] B_{\ell}(j)[b]\right) \quad(\bmod 2) \\
& =\sum_{q \in[\ell] \backslash\{b\}} B_{\ell}(i)[q] B_{\ell}(j)[q] \quad(\bmod 2), \quad \text { since } B_{\ell}(j)[b]=0 \\
& =c_{\phi_{b}(i)}\left[\phi_{b}(j)\right] .
\end{aligned}
$$

It remains to show there is a bijection between the elements of $[(k+1) / 2-1]$ and the corresponding elements of $[k]$. For each $i \in[(k+1) / 2-1]$, there are exactly two distinct strings $i^{\prime}, i^{\prime \prime} \in[k]$ in the preimage of $\phi_{b}(i)$ whose length- $\ell$ bitstring representations differ only by the bit in the $b$-th position. Without loss of generality, suppose $B_{\ell}\left(i^{\prime}\right)[b]=1$. We claim that either $c_{i^{\prime}}$ or $c_{i^{\prime \prime}}$ is in the orthogonal subcode. If $c_{i^{\prime}} \in \mathscr{C}_{\boldsymbol{z}}^{\perp}(\ell)$, then the claim is shown, otherwise, suppose $c_{i^{\prime}}$ is not in the orthogonal subcode. Then

$$
\begin{aligned}
0 & \neq \sum_{r=1}^{k} c_{i^{\prime}} z[r]=c_{i^{\prime}}[a] \\
& =\sum_{q=1}^{\ell} B_{\ell}\left(i^{\prime}\right)[q] B_{\ell}(a)[q] \\
& =\sum_{q \in[\ell] \backslash\{b\}} B_{\ell}\left(i^{\prime}\right)[q] B_{\ell}(a)[q]+B_{\ell}(a)[b]
\end{aligned}
$$

since $B_{\ell}\left(i^{\prime}\right)[b]=1$. Since $B_{\ell}\left(i^{\prime \prime}\right)[b]=0$, and the strings only differ in the $b$-th position, changing the $i$-th bit to zero changes the parity of the sum, and we conclude that $c_{i^{\prime \prime}} \in \mathscr{C}_{z}^{\perp}$. Finally, it is easy to see that each $j \in[k]$ with $B_{\ell}(j)[b]=0$ corresponds to exactly one element of $[(k+1) / 2-1]$.

Let $\widehat{\boldsymbol{y}} \in \mathbb{R}^{(k+1) / 2-1}$ be the vector constructed from $\boldsymbol{y}$ as $\widehat{\boldsymbol{y}}\left[\phi_{b}(j)\right]=$ $\boldsymbol{y}[j]$ for each $j \in[k]$ such that $B_{\ell}(j)[b]=0$. Thus $\widehat{\boldsymbol{y}}$ is obtained from $\boldsymbol{y}$ by removing the indexes of $\boldsymbol{y}$ in which the bitstring representation of the index contains a 1 in the $b$-th position. It is easy to see that if $\boldsymbol{y}$ is orthogonal to $c_{i} \in \mathscr{C}_{\boldsymbol{z}}^{\perp}(\ell)$, then $\widehat{\boldsymbol{y}}$ is necessarily orthogonal to $c_{\phi_{b}(i)} \in \mathscr{C}(\ell-1)$, since orthogonality is preserved by index deletion. Moreover, $|\operatorname{supp}(\widehat{\boldsymbol{y}})| \leq|\operatorname{supp}(\boldsymbol{y})|$ since no nonzero elements are created under the transformation.

Then $\boldsymbol{x}=\boldsymbol{y}+\boldsymbol{z}$ is orthogonal to the code words in the orthogonal subcode $\mathscr{C}_{\boldsymbol{z}}^{\perp}(\ell)$ that are also orthogonal to $\boldsymbol{y}$. By induction, $\widehat{\boldsymbol{y}} \in$ $\mathbb{R}^{(k+1) / 2-1}$ is orthogonal to at least $2^{(\ell-1)-|\operatorname{supp}(\widehat{\boldsymbol{y}})|}-1$ code words in $\mathscr{C}(\ell-1)$. By the congruence between $\mathscr{C}_{z}^{\perp}(\ell)$ and $\mathscr{C}(\ell-1)$, and since $|\operatorname{supp}(\widehat{\boldsymbol{y}})| \leq|\operatorname{supp}(\boldsymbol{y})|, \boldsymbol{y}$ is orthogonal to at least

$$
2^{(\ell-1)-|\operatorname{supp}(\boldsymbol{y})|}-1=2^{\ell-|\operatorname{supp}(\boldsymbol{x})|}-1
$$

code words in $\mathscr{C}_{\boldsymbol{z}}^{\perp}(\ell) \subseteq \mathscr{C}(\ell)$. Finally, all $2^{\ell}-1$ code words in $\mathscr{C}(\ell)$ are orthogonal to the zero vector, which fulfills the base case, completing the proof. 